\title{
The Importance of Haemogram Parameters in the Diagnosis and Prognosis of Septic Patients
}

\author{
Alina Elena Orfanu1,2, Cristina Popescu1,2, Anca Leuștean ${ }^{1 *}$, Anca Ruxandra Negru1,2, \\ Cătălin Tilişcan ${ }^{1,2}$, Victoria Aramă1,2, Ștefan Sorin Aramă1,2 \\ ${ }^{1}$ National Institute for Infectious Diseases "Prof. Dr Matei Balș”, Dr Calistrat Grozovici Street, no 1, 021105, \\ Bucharest, Romania \\ 2 University of Medicine and Pharmacy "Carol Davila”, Dionisie Lupu Street, no 37, 020021, Bucharest, Romania
}

\begin{abstract}
Sepsis represents a severe pathology that requires both rapid and precise positive and differential diagnosis to identify patients who need immediate antimicrobial therapy. Monitoring septic patients' outcome leads to prolonged hospitalisation and antibacterial therapy, often accompanied by substantial side effects, complications and a high mortality risk.

Septic patients present with complex pathophysiological and immunological disorders and with a predominance of pro-inflammatory or anti-inflammatory mediators which are heterogeneous with respect to the infectious focus, the aetiology of sepsis or patients' immune status or comorbidities. Previous studies performed have analysed inflammatory biomarkers, but a test or combinations of tests that can quickly and precisely establish a diagnosis or prognosis of septic patients has yet to be discovered. Recent research has focused on re-analysing older accessible parameters found in the complete blood count to determine the sensitivity, specificity, positive and negative predictive values for the diagnosis and prognosis of sepsis.

The neutrophil/lymphocyte count ratio (NLCR), mean platelet volume (MPV) and red blood cells distribution width (RDW) are haemogram indicators which have been evaluated and which are of proven use in septic patients' management.
\end{abstract}

Keywords: neutrophil/lymphocyte count ratio, mean platelet volume, red blood cells distribution width, sepsis

Received: 4 May 2017 / Accepted: 11 July 2017

\section{INTRODUCTION}

Sepsis is a clinical syndrome whose incidence and mortality rate has increased over the last thirty years, [1] as a consequence of patients' advanced age, immunosuppressive diseases and therapies or infections with multi-drug resistant bacteria [2]. It remains the leading cause of death in non-coronary intensive care units worldwide, with a mortality rate estimated at $30 \%$ in sepsis and $80 \%$ in septic shock in the USA [3] and at $12.8 \%$ in sepsis and $45.7 \%$ in septic shock in Europe [4]. Reduced rates of reporting may affect estimations in developing countries.

The concept of sepsis, defined for the first time at the beginning of the 90's and updated in 2001 [5], refers to the presence of an infectious systemic inflammatory response syndrome (SIRS). The definition was reanalysed and modified in 2016 relating it to a severe organ dysfunction, caused by an inadequate response of the organism to an infectious agent [6]. The prognosis and the rate of mortality are now estimated using the SOFA score (The Sequential Organ Failure Assessment score). This definition, nevertheless, does not offer clear criteria for sepsis diagnosis. In this context, sepsis raises multiple problems of diagnosis and prognosis, and further studies are necessary to identify useful criteria for establishing a rapid and correct diagnosis and quick and effective treatment.

Several inflammatory biomarkers have been evaluated with the aim of identifying those with the highest 
sensitivity, specificity, positive and negative predictive values for sepsis diagnosis. Another problem is the assessment of the septic patient outcome during treatment as currently used clinical and biological criteria are undefined and inadequate for this purpose.

Previously published studies have investigated the use of inflammatory biomarkers in assessing the severity and outcome of the disease treated under defined antimicrobial therapy. They tend to offer little precise results due to group heterogeneity and the inclusion of patients with various comorbidities.

\section{SEARCH CRITERIA}

There is an extensive literature regarding inflammatory biomarkers in sepsis. These include haemogram indicators like Neutrophil/Lymphocyte Count Ratio (NLCR), Mean Platelet Volume (MPV), Red Blood Cells Distribution Width (RDW), and continue with the classic inflammatory tests such as fibrinogen, Creactive protein (CRP) and go on with the discovery of procalcitonin (PCT), soluble-CD14 subtype (sCD14ST, presepsin), pro-adrenomedullin (pro-ADM), soluble triggering receptor expressed on myeloid cells-1 (sTREM-1), interleukin-6 (IL6), interleukin-8 (IL8), interleukin-27 (IL27), soluble urokinase-type plasminogen activator receptor (suPAR).

To date, no inflammatory biomarker has been identified which exhibits a high degree of sensitivity and specificity which would permit the identification of patients who need rapid antibiotic therapy.

The literature focuses on NLCR, MPV and RDW, i.e., the three haemogram parameters which are easy to evaluate, and which do not incur additional costs to routine analysis.

\section{- LITERATURE REVIEW}

Neutrophil/lymphocytes count ratio (NLCR) has been the focus of several recent studies published as it is accessible, cheap and readily determined. The importance of this parameter is related to the pathophysiological mechanism of SIRS, which is characterised by an increased number of circulating leucocytes, due to the increased number of neutrophils, the first line of antimicrobial defence. On the other hand, lymphocytopenia appears as a consequence of lymphocyte margination and redistribution in the lymphatic system, with accelerated apoptosis [7].
The predictive role of NLCR has been evaluated not only in septic patients but also in patients with tumours, cardiovascular diseases or intestinal inflammatory diseases. The initial value of this indicator was correlated with the outcome and with the survival rate in patients with different types of cancers: pulmonary, breast, prostatic, pancreatic, oesophageal [8], colorectal [9] or hepatocellular carcinoma followed by liver transplant [10]. Several studies, published in cardiovascular medicine domain, showed the prognostic role of NLCR in patients with an acute coronary syndrome, aortocoronary bypass or congestive heart failure [11, 12]. Moreover, Ertas et al. (2013) indicated that NLCR was useful in the prognosis of patients with thromboembolic stroke [13]. On the other hand, a high correlation between NLCR and the outcome of patients with gangrenous appendicitis was demonstrated, and was shown to be superior to other parameters like fever, CRP, Leucocytes number or the Glasgow scale [14].

Moreover, NLCR is considered to be superior to other biomarkers such as CRP, leukocytes count or neutrophils count, as a predictor for bacteraemia in patients admitted to emergency or intensive care units [15]. In a study of 40 patients with severe sepsis, Okashah et al. (2014) highlighted NLCR superiority regarding sensitivity, specificity, positive and negative predictive values to other parameters like lactate, CRP, neutrophils count, lymphocytes count, or leucocytes count. [16] The same study showed the usefulness of NLCR in prognostic evaluation by highlighting the statistically significant correlation with two severity scores, Acute Physiology and Chronic Health Evaluation (APACHE) II $(\mathrm{p}=0.01)$ and SOFA $(\mathrm{p}=0.01)$.

Other studies attempted to establish threshold values for NLCR to predict the severity and outcome of the disease. A retrospective study, which included 2311 patients with bacteraemia concluded that a value of NLCR over seven on admission, represented an independent increased mortality rate risk factor [17]. A more recent study showed that an initial value of NLCR over ten could be correlated with an unfavourable prognosis, as assessed by the number of SIRS criteria, the presence of organ failures or septic metastasis at admission. Despite the low number of investigated patients, this study tended to substantiate the prognostic role of NLCR in sepsis through the statistically significant correlations with APACHE IV $(p=0.01)$ and APS $(p=0.01)$ scores and with the estimated rate of mortality $(\mathrm{p}=0.01)$ [18]. 
Mean platelet volume (MPV) is another haemogram parameter which has gained importance in the management of septic patients. Sepsis induces multiple changes in the organism systems, including the haemostasis, platelets activation including excessive aggregation and destruction, with consequences on their number and dimension. Platelet activation is caused by endothelial injuries triggered by the infectious agent. In this context, an increased MPV and a reduced platelet count are associated with an unfavourable prognosis and with an elevated mortality risk [19].

Studies published in the early 80's showed elevated values of MPV in about half of patients with systemic infection, in contradistinction to those with localised bacterial infections and negative blood cultures. In these cases, MPV values remained within normal limits, independent of platelet count. Same authors concluded that in patients who received effective antimicrobial therapy, MPV became normal within one week. Also, the increase of MPV in patients with localised infection was considered a signal for a generalised infection [20].

The further discovery of other inflammatory biomarkers tests with a superior sensitivity such as fibrinogen, CRP or PCT, lead to MPV being marginalised form the core literature. However, recently, numerous cost-effective studies have been published which feature this accessible parameter. One study involving 183 septic patients revealed a correlation between MPV value at admission and the risk of death. Patients who died presented with a higher initial value of MPV ( 9.6 versus $9.19 \mathrm{fl}, \mathrm{p}=0.031$ ) [21]. The relation between MPV and the disease severity as evaluated by APACHE IV, and APS scores, were also shown to be statistically significant ( $\mathrm{p}=0.03$, respective $\mathrm{p}=0.02)$ [22].

Establishing a threshold value for the diagnosis or prognosis of sepsis has been the target of several recent studies. A level of MPV over eight $\mathrm{fl}$ is associated with moderate sensitivity (53.47\%) and excellent diagnostic specificity. Besides thrombocytopenia and elevated MPV, an increased platelet distribution width (PDW) was also registered in septic patients, and it was found that a value over $18 \%$ is associated with a higher risk of death [23].

The predictive value of MPV in appreciating the mortality risk was not demonstrated in patients with septic shock, in which MPV median was similar between the group of deceased patients and the survivor group (10.6 $\mathrm{fl}[ \pm 0.9]$ versus $10.5 \mathrm{fl}[ \pm 0.9]$. In this study, other parameters such as age, APACHE and SOFA scores and the platelet count indicated significant differences between the two groups of patients [24].

MPV was evaluated for neonatal sepsis prognosis in multiple studies in which a comparative analysis was made between patients with proven sepsis and a healthy control group. The evaluation of inflammatory biomarkers at admission revealed higher values of the leucocyte count, CRP, IL- 6 and MPV in patients with sepsis $(\mathrm{p}=0.01, \mathrm{p}<0.001, \mathrm{p}<0.001$, respectively $\mathrm{p}=0.001$ ), compared to the control group. Furthermore, the CRP and MPV medians were significantly higher in patients with positive microbiological tests, comparative to patients with sepsis of unknown aetiology [25]. MPV proved a good predictor of the outcome of children with community acquired pneumonia, but with limited specificity and a negative predictive value and a high rate of false-negative results [26].

MPV has been shown to be useful in the prognosis of other non-infectious pathologies, such as ischemic heart disease [27] or stroke [28], as much as other markers of unspecific inflammation.

Red cell distribution width (RDW) represents an indicator which can vary in sepsis, under the influence of pro-inflammatory cytokines (TNF $\alpha$, IFN $\delta$, IL-1 $\beta$, IL-6) [29], released during the inflammatory process. These cytokines cause inefficient erythropoiesis resulting in structural and functional changes of erythrocytes, with volume variations and increased RDW. High values of this parameter can also appear in nutritional deficiencies such as iron deficiency anaemia, vitamin B12 or folate deficiency anaemia, or in blood transfusions [30]. As RDW is a marker of unspecific inflammation, it can increase in many other diseases such as heart failure, stroke, peripheral arterial disease or chronic pulmonary diseases [31].

Recent studies have centred on evaluating RDW's prognostic value and less for the diagnostic role in sepsis. A study, which enrolled 349 patients, revealed the function of RDW in appreciating septic patients' outcome through the statistically significant association with APACHE II score and with hospital mortality rate. $(\mathrm{p}<0.0001)$. This study demonstrated that an RDW value over $16 \%$ is concurrent with a higher APACHE value and risk of death [31]. Kim et al. (2015) evaluated the predictive role of RDW regarding the short and medium-term mortality in elderly patients with severe sepsis and septic shock and concluded that every one percent (1\%) increase in RDW is equivalent to a 
$15 \%$ increase in the mortality rate in the first 30 days [32]. RDW was also considered a good predictor of the length of hospitalisation for septic patients admitted to intensive care units [33].

This biomarker proved its usefulness as an outcome measure because an ascending trend of RDW in the first 72 hours of admission is associated with an unfavourable prognosis, even though the initial values were normal [34]. Chen et al. (2015) analysed almost 7000 adult patients with sepsis, with a mortality rate of $6.8 \%$. In this study, patients who died had higher initial values of RDW than the survivors $(15.7 \%$ versus $13.8 \%)$. There were also established threshold values; for RDW over than $15.6 \%$, the risk of death was $16.7 \%$, for RDW between $14-15.6 \%$ it was $7.3 \%$, and for RDW under $13.1 \%$ the mortality rate was $1.6 \%$. Thus, RDW was considered to be a superior mortality predictive factor to SIRS criteria, the MEDS (Mortality in Emergency Department Sepsis) or CURB65 scores [35]. Similar results were published by Lorente et al. (2014) showing that RDW is a low-cost procedure which should be routinely performed to identify the risk of death in sepsis. In this study, the prognostic value of RDW was compared with other biomarkers such as serum malondialdehyde and $\alpha \mathrm{TNF}$, with comparable results [36].

\section{DISCUSSION}

Sepsis pathogenesis is complex, with heterogeneous pathophysiological and immunological variations related to the aetiology, primary septic focus, organ dysfunctions and pre-existent pathologies. Another problem is the differential diagnosis between an infectious and a non-infectious cause of SIRS because a delay in initiating antimicrobial therapy in a septic patient can significantly increase the risk of death.

The microbiological examination remains the gold standard for the diagnosis of sepsis, and it is preferable because it identifies the incriminating bacterial pathogens and allows the administration of an appropriate antimicrobial regimen, according to an antibiogram. However, both the prognostic and monitoring role of microbiological tests are limited. On the other hand, it can be influenced by several factors such as the ability of the bacteria to grow in cultures, the site of the infectious focus, the antibiotics received before the tests or the equipment of each laboratory. Concerning the prognosis, sepsis is a life-threatening syndrome, responsible for multiple complications, several organ failures and high mortality rate.

Within this context, additional studies to identify parameters useful for the diagnosis and prognosis of septic patients are required. Current published data are controversial because they include multiple biomarkers with different sensitivity, specificity, positive or negative predictive values and have been studied using heterogeneous patient groups. Further research of these biomarkers may provide valuable data and suggest which markers can be used to establish a rapid positive and differential diagnosis between infectious and noninfectious SIRS, how these markers can be correlated with the severity of sepsis or how its dynamics can predict the septic patient outcome.

At present biomarkers like CRP, IL-6 and PCT are frequently used in diagnosing and monitoring patients with sepsis. CRP is involved in innate immune system regulation and represents a highly sensitive acute phase reactant, but with limited specificity for infectious systemic inflammatory response syndrome. This biomarker is usually associated with chronic inflammatory processes such as autoimmune or rheumatological disorders, haematological diseases or tumours. Recent studies which evaluated different inflammatory biomarkers showed that CRP is inferior to PCT and NLCR, regarding sensitivity ( $80 \%$ versus $91.2 \%$ and $88 \%$ respectively), specificity (65\% versus $82 \%$ and $75 \%$ respectively), positive ( $82 \%$ versus $90 \%$ and $87.5 \%$ respectively) and negative (66.5\% versus $80 \%$ and $75 \%$ respectively) predictive values [16].

IL-6, a cytokine released from the inflammatory process along with IL- $1 \beta$ or TNF $\alpha$, represents another useful parameter to guide the clinician for the diagnosis and prognosis of sepsis. Jekarl et al. (2013) found that IL- 6 is a better test than CRP and PCT in estimating bacterial sepsis severity. In this study, $86 \%$ of survivors registered a rapid decrease of IL- 6 values in contrast with the others [37]. Klag et al. (2016) held the same opinion concerning the use of IL- 6 in assessing the severity of the condition. In the first $24-48$ hours, levels of IL-6 declined steeply in patients who survived contrary to non-survivors and had a higher sensitivity than other inflammatory markers [38].

Arguably, PCT is the most specific test for bacterial sepsis though it is only moderate with respect to sensitivity. Recent studies consider that PCT has higher specificity for sepsis than presepsin or other new sophisticated parameters [39]. Several authors concluded 
that in comparison to the other haemogram indicators reviewed in this article, PCT has superior sensitivity, specificity and predictive values, but with minor differences. For example, Zhang et al. (2016) indicated that PCT and NLCR have similar predictive values for sepsis [40].

Whereas some authors are interested in the discovery of new cytokines which can be used as inflammatory biomarkers, numerous recent studies have focused on the seeing afresh older inexpensive tests.

This literature review emphasises the importance of three haemogram parameters (NLCR, MPV, RDW) which can help the clinician to arrive at a prompt diagnosis and to monitor septic patients' outcome.

\section{- AUTHORS' CONTRIBUTIONS STATEMENT}

The authors have equally contributed to the writing of this work.

\section{- CONFLICTS OF INTEREST}

The authors declare no conflicts of interest.

\section{ACKNOWLEDGMENT}

This paper is part of "Carol Davila" doctoral programme.

\section{REFERENCES}

1. Cho WH. Update of Sepsis: Recent Evidences about Early Goal Directed Therapy. Tuberc Respir Dis (Seoul). 2015;78(3):156160.

2. Esper AM, Martin GS. Extending international sepsis epidemiology: the impact of organ dysfunction. Crit Care. 2009;13(1):120.

3. Martin GS. Sepsis, severe sepsis and septic shock: changes in incidence, pathogens and outcomes. Expert Rev Anti Infect Ther. 2012;10(6):701-6.

4. Esteban A, Frutos-Vivar F, Ferguson ND, et al. Sepsis incidence and outcome: contrasting the intensive care unit with the hospital ward. Crit Care Med. 2007;35(5):1284-9.

5. Levy MM, Fink MP, Marshall JC, et al. 2001 sccm/esicm/accp/ ats/sis international sepsis definitions conference. Intensive Care Med. 2003;29(4):530-8.

6. Shankar-Hari M, Phillips GS, Levy ML, et al. Developing a new definition and assessing new clinical criteria for septic shock: for the Third International Consensus Definitions for Sepsis and Septic Shock (Sepsis-3). JAMA. 2016;315(8):775-87.
7. Joshi VD, Kalvakolanu DV, Cross AS. Simultaneous activation of apoptosis and inflammation in pathogenesis of septic shock: a hypothesis. FEBS Lett. 2003;555(2):180-4.

8. Templeton AJ, McNamara MG, Šeruga B, et al. Prognostic role of neutrophil-to-lymphocyte ratio in solid tumors: a systematic review and meta-analysis. J Natl Cancer Inst. 2014;106(6):dju124.

9. Walsh SR, Cook EJ, Goulder F, Justin TA, Keeling NJ. Neutrophillymphocyte ratio as a prognostic factor in colorectal cancer. J Surg Oncol. 2005;91(3):181-4.

10. Halazun KJ, Hardy MA, Rana AA, et al. Negative impact of neutrophil-lymphocyte ratio on outcome after liver transplantation for hepatocellular carcinoma. Ann Surg 2009;250(1):141-51.

11. Tamhane UU, Aneja S, Montgomery D, Rogers EK, Eagle KA, Gurm HS. Association between admission neutrophil to lymphocyte ratio and outcomes in patients with acute coronary syndrome. Am J Cardiol 2008;102(6):653-7.

12. Gibson $\mathrm{PH}, \mathrm{Croal} \mathrm{BL}$, Cuthbertson $\mathrm{BH}$, et al. Preoperative neutrophil-lymphocyte ratio and outcome from coronary artery bypass grafting. Am Heart J. 2007;154(5):995-1002.

13. Ertaş G, Sönmez O, Turfan M, et al. Neutrophil/lymphocyte ratio is associated with thromboembolic stroke in patients with non-valvular atrial fibrillation. J Neurol Sci. 2013;324(1):49-52.

14. Ishizuka M, Shimizu T, Kubota K. Neutrophil-to-lymphocyte ratio has a close association with gangrenous appendicitis in patients undergoing appendectomy. Int Surg. 2013;97(4):299304.

15. de Jager CP, van Wijk PT, Mathoera RB, de Jongh-Leuvenink J, van der Poll T, Wever PC. Lymphocytopenia and neutrophillymphocyte count ratio predict bacteremia better than conventional infection markers in an emergency care unit. Crit care. 2010;14(5):R192.

16. Okashah AS, El-Sawy MM, Beshay BN, Abd El-Raouf A. Ratio of Neutrophil to Lymphocyte counts as a simple marker for sepsis and severe sepsis in Intensive Care Unit. Res Opin Anesth Intensive Care. 2014;2:39-45.

17. Terradas R, Grau S, Blanch J, et al. Eosinophil count and neutrophil-lymphocyte count ratio as prognostic markers in patients with bacteremia: a retrospective cohort study. PloS One. 2012;7(8):e42860.

18. Orfanu A, Aramă V, Aramă ŞS, et al. The diagnostic and prognostic role of neutrophil to lymphocyte count ratio in sepsis. BMC Infect Dis. 2016;16(4):A5.

19. Zampieri FG, Ranzani OT, Sabatoski $V$, et al. An increase in mean platelet volume after admission is associated with higher mortality in critically ill patients. Ann Intensive Care. 2014;4:20.

20. Van der Lelie J, Von dem Borne AK. Increased mean platelet volume in septicaemia. J Clin Pathol. 1983;36(6):693-6.

21. Eberhardt A, Lessig F, Schreiter K, et al. Mean platelet volume (MPV) is an outcome marker in sepsis patients. Int J Infect Dis. 2012;16:e218. 
110 The Journal of Critical Care Medicine 2017;3(3)

22. Orfanu A, Popescu C, Leustean A, et al. The evaluation of the prognostic value of procalcitonin, mean platelet volume and neutrophil/lymphocytes ratio in sepsis. The Scientific Days of the National Institute of Infectious Diseases "Prof. Dr. Matei Bals". Abstract Volume - ID in ID or Identity Definition in Infectious Diseases 2015;17.

23. Guclu E, Durmaz Y, Karabay O. Effect of severe sepsis on platelet count and their indices. Afr Health Sci. 2013;13(2):333-8.

24. Sadaka F, Donnelly PL, Griffin MT, Brien JO, Lakshmanan R. Mean Platelet Volume is not a Useful Predictor of Mortality in Septic Shock. Blood Disorders Transf. 2014;5:2.

25. Oncel MY, Ozdemir R, Yurttutan S, et al. Mean Platelet Volume in Neonatal Sepsis. J Clin Lab Anal. 2012;26(6):493-6.

26. Karadag-Oncel E, Ozsurekci Y, Kara A, Karahan S, Cengiz $A B$, Ceyhan M. The value of mean platelet volume in the determination of community acquired pneumonia in children. Ital J Pediatr. 2013;39(1):16.

27. Ki YJ, Park S, Ha SI, Choi DH, Song H. Usefulness of mean platelet volume as a biomarker for long-term clinical outcomes after percutaneous coronary intervention in Korean cohort: a comparable and additive predictive value to high-sensitivity cardiac troponin $\mathrm{T}$ and $\mathrm{N}$-terminal pro-B type natriuretic peptide. Platelets. 2014;25(6):427-32.

28. D'erasmo E, Aliberti G, Celi FS, Romagnoli E, Vecci E, Mazzuoli GF. Platelet count, mean platelet volume and their relation to prognosis in cerebral infarction. J. Intern. Med. 1990;227(1):11-4.

29. Scharte M, Fink MP. Red blood cell physiology in critical illness. Crit Care Med. 2003;31(12):S651-7.

30. Fukuta H, Ohte N, Mukai S, et al. Elevated plasma levels of B-type natriuretic peptide but not C-reactive protein are associated with higher red cell distribution width in patients with coronary artery disease. Int Heart J. 2009;50(3):301-12.

31. Mahmood NA, Mathew J, Kang B, DeBari VA, Khan MA.
Available online at: www.jccm.ro

Broadening of the red blood cell distribution width is associated with increased severity of illness in patients with sepsis. Int J Crit IIIn Inj Sci. 2014;4(4):278-282.

32. Kim S, Lee K, Kim I, Jung S, Kim MJ. Red cell distribution width and early mortality in elderly patients with severe sepsis and septic shock. Clin Exp Emerg Med. 2015;2(3):155-161.

33. Wang F, Pan W, Pan S, Ge J, Wang S, Chen M. Red cell distribution width as a novel predictor of mortality in ICU patients. Ann. Med. 2011;43(1):40-6.

34. Kim CH, Park JT, Kim EJ, et al. An increase in red blood cell distribution width from baseline predicts mortality in patients with severe sepsis or septic shock. Crit Care. 2013;17(6):R282.

35. Chen CK, Lin SC, Wu CC, Chen LM, Tzeng IS, Chen KF. STARDcompliant article: The utility of red cell distribution width to predict mortality for septic patients visiting the emergency department. Medicine. 2016;95(24):e3692.

36. Lorente L, Martín MM, Abreu-González P, et al. Red blood cell distribution width during the first week is associated with severity and mortality in septic patients. Plos One. 2014;9(8):e105436.

37. Jekarl DW, Lee SJ, Lee J, et al. Procalcitonin as a diagnostic marker and IL-6 as a prognostic marker for sepsis. Diagn Microbiol Infect Dis 2013;75:342-7.

38. Klag T, Cantara G, Sechtem U, Athanasiadis A. Interleukin-6 kinetics can be useful for early treatment monitoring of severe bacterial sepsis and septic shock. Infect Dis Rep. 2016; 8(1):6213.

39. Henriquez-Camacho C, Losa J. Biomarkers for sepsis. BioMed research international. 2014;2014:547818.

40. Zhang HB, Chen J, Lan QF, Ma XJ, Zhang SY. Diagnostic values of red cell distribution width, platelet distribution width and neutrophillymphocyte count ratio for sepsis. Exp Ther Med. 2016;12(4):2215-9. 